\title{
A General Framework for Modular Implementations of Modular System Specifications
}

\author{
Michel Bidoit*, Rolf Hennicker** \\ *LIENS, CNRS \& Ecole Normale Supérieure \\ 45, Rue d'Ulm, 75230 Paris Cedex, FRANCE \\ **Institut für Informatik, Ludwig-Maximilians-Universität München \\ Leopoldstr. 11b, D-8000 München 40, GERMANY
}

\begin{abstract}
We investigate the impact of modularity on the semantics and on the implementation of software specifications. Based on the stratified loose semantics approach we develop a suitable specification framework which meets our basic requirements: the independent construction of implementations for the single constituent parts (modules) of a system specification and the encapsulated development of each implementation part using the principle of stepwise refinement.

Our paper is not aimed at providing an elaborated specification language but rather to concentrate on the modularity issues of system development. Hence, only few but powerful constructs are provided which can be seen as a kernel for further extensions. In particular, we will show that implementation and parameterization can be handled within a uniform concept and we will prove compatibility theorems like the horizontal composition property. All constructs are defined on top of a very general logical framework thus being applicable to various kinds of specification formalisms.
\end{abstract}

\section{Introduction}

The modular design of software systems has obvious advantages: it increases the understandability of a software system, supports the reuse of single modules in different applications and allows the independent realization of modules by different programmer teams. But not only the modular design is important: It is crucial that each implementation of a single module meets its requirements. Hence a safe development methodology is needed which allows to split the construction of a single implementation task into well defined steps, each step being manageable more easily than an ad hoc construction of a realization from scratch. This is commonly called the principle of stepwise refinement.

In this paper we will develop a suitable specification framework which allows both, the independent construction of implementations for the single constituent parts (modules) of a system specification and the separate development of single implementations by stepwise refinement. We set out from the stratified loose semantics approach of [Bidoit 87] which reflects the modular properties of a specification at the semantical level. In particular, only those realizations of a system specification are accepted which are constructable by the "composition" of single realizations, each single realization being compatible with arbitrary implementations of the other constituent parts of the specification. As a consequence we use a clean distinction between specification modules and their associated system specifications: modules are interpreted by classes of functors while system specifications are interpreted by classes of algebras.

In [Bidoit 87] implementations are treated at the semantical level as models or functors of a specification (module). Hence it is not possible to deal with the development of implementations by stepwise refinements of their abstract 
specifications. In the present paper we overcome this problem by introducing an implementation relation for system specifications and for specification modules which is based on the stratified loose semantics. Due to the loose semantics approach the implementation -definition is straightforward by requiring that an implementing module restricts the class of possible realizations (functors) of an abstract specification module. An analogous definition is used for implementations of system specifications considering algebras instead of functors. Since implementations are now handled at the specification level we have provided a suitable basis for the development of formal proof calculi for correctness proofs but this is not in the scope of this paper.

It turns out that our implementation definition has exactly all the properties required for modular system design: it allows independent implementations of modules and sub-systems, it ensures that the properties of "local" implementations are preserved by global ones and it supports the reusability of existing implementations in different contexts. In particular, it guarantees that exchanging a sub-system implementation by another one still produces a globally correct system implementation.

Usually implementations are built on top of already existing specifications of standard data structures (such as arrays, lists, trees etc.). Hence, in general, implementation relations will be established by connecting the signatures of abstract and concrete system specifications (modules resp.) by a signature morphism which maps, if we consider algebraic signatures, abstract sorts and operations to their concrete representations. This shows that there is a close relationship between implementation and parameter actualization (in the classical sense of parameterized specifications). Indeed, in the loose semantics approach, not only syntactically but even semantically there is conceptually no difference between implementation and parameter actualization since in both cases the requirements of an abstract specification, a formal parameter specification resp., must be satisfied by an implementation, by an actual parameter resp.. Hence, in our approach, sub-system specifications can be seen as formal parameters and the implementation of sub-system specifications can be viewed as actualization (parameter passing). As a consequence we deal with both aspects in one uniform framework and the various correlations between implementation and parameterization will be studied using the same formal techniques.

This indicates already that our aim is not the development of an elaborated specification language with a couple of syntactic concepts. On the contrary, we want to concentrate ourselves on the investigation of modularity and implementation issues using only a few but powerful constructs. For the same reason, our approach is developed within a very general logical framework such that it can be instantiated by a series of particular specification formalisms.

\section{Assumptions on the Underlying Logical Framework}

The approach on modular system design presented in this paper works in a very general setting. In this section we will summarize our assumptions on the underlying logical framework. Note that we do not intend to propose a new powerful framework as an alternative to institutions or specification logics. On the contrary, we will see that we can use such frameworks as a particular basis for our approach. What we need is:

1. A category SIGN of signatures which has inclusions and pushouts [Schoett 87]. We assume that SIGN has an initial object $\emptyset$, called empty signature. If $\Sigma, \Sigma^{\prime} \in$ SIGN and t: $\Sigma \rightarrow \Sigma^{\prime}$ is an inclusion morphism we also write $\Sigma \subseteq \Sigma^{\prime}$. Pushout diagrams are of the 
form

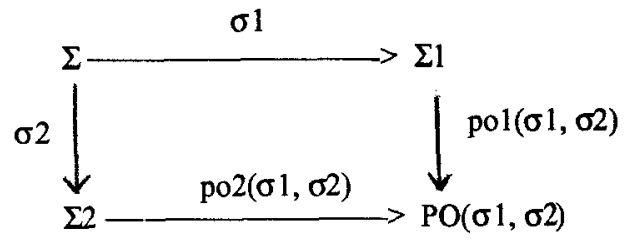

2. A functor Alg: SIGN $\rightarrow$ CAT ${ }^{\text {op }}$ where CAT is the category of all categories. For any signature morphism $\sigma: \Sigma \rightarrow \Sigma^{\prime}$ the functor $\operatorname{Alg}(\sigma): \operatorname{Alg}\left(\Sigma^{\prime}\right) \rightarrow \operatorname{Alg}(\Sigma)$ is called forgetful functor associated to $\sigma$. It will be denoted by $U_{\sigma}$ (or simply by $U$ if $\sigma$ is clear from the context).

The functor Alg translates any pushout diagram into the following diagram in CATop:

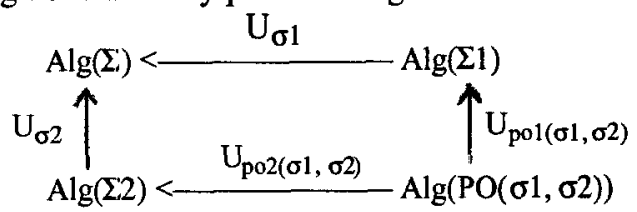

We assume that any pushout diagram in SIGN as given above has amalgamations w.r.t. Alg. In particular this means that for any two objects $A 1 \in \operatorname{Alg}(\Sigma 1), A 2 \epsilon$ $\operatorname{Alg}(\Sigma 2)$ with $\mathrm{U}_{\sigma 1}(\mathrm{~A} 1)=\mathrm{U}_{\sigma 2}(\mathrm{~A} 2)$ there exists a unique object $\mathrm{A} 2 \oplus \mathrm{Al} \in \mathrm{Alg}(\mathrm{PO}(\sigma 1$, $\sigma 2)$ ) such that $U_{\mathrm{po} 2(\sigma 1, \sigma 2)}(\mathrm{A} 2 \oplus \mathrm{A} 1)=\mathrm{A} 2$ and $U_{\mathrm{po} 1(\sigma 1, \sigma 2)}(\mathrm{A} 2 \oplus \mathrm{A} 1)=\mathrm{A} 1$. For $\mathrm{a}$ precise definition of amalgamations see e.g. [Ehrig et al. 89], [Ehrig et al. 91].

Lemma 1 Assume that a pushout diagram is given as above.

If $\mathrm{M} \subseteq \mathrm{Alg}(\Sigma)$ and $\mathrm{M} 2 \subseteq \operatorname{Alg}(\mathbb{D})$ are subcategories such that $\mathrm{U}_{\sigma 2}(\mathrm{M} 2) \subseteq \mathrm{M}$ and if

$\mathrm{F}: \mathrm{M} \rightarrow \operatorname{Alg}(\Sigma 1)$

is a functor such that $U_{\sigma 1}(F(A))=A$ for all $A \in M$ then there exists a unique functor

$\mathrm{F}^{\prime}: \mathrm{M} 2 \rightarrow \mathrm{Alg}(\mathrm{PO}(\sigma 1, \sigma 2))$

such that $F^{\prime}(A 2)=A 2 \oplus F\left(U_{\sigma 2}(A 2)\right)$. $F^{\prime}$ is called extension of $F$ w.r.t. $\sigma 2$.

Proof: The proof can be done by a slight generalization of the extension lemma (cf. e.g. [Ehrig et al. 89]).

3. Finally we assume a class SPEC of specifications and two functions Sig: SPEC $\rightarrow$ SIGN and Mod: SPEC $\rightarrow$ CATop such that for all SP $\in$ SPEC, Mod(SP) is a subcategory of $\mathrm{Alg}(\mathrm{Sig}(\mathrm{SP}))$. The objects of $\mathrm{Mod}(\mathrm{SP})$ are called models of SP.

Examples 1. All specifications constructed in an arbitrary institution (c.f. [Goguen, Burstall 84]) by the specification building primitives of [Sannella, Tarlecki 84] can be used as the underlying specification language SPEC of our approach if pushouts, inclusions and amalgamations exist. In particular, semiexact reasonable institutions as defined in [Diaconescu et al. 91] provide a suitable basis.

2. The behavioural specification approaches of [Bernot, Bidoit 91] and [Hennicker 92] can be used as a basis for the present framework although they do not provide an institution.

3. Let (ASPEC, Catmod) be a specification logic in the sense of [Ehrig et al. 89] which has pushouts and amalgamations. Moreover, let SIGN be a subcategory of ASPEC which has inclusions and is closed under pushouts and let Sig: ASPEC $\rightarrow$ SIGN be a function such that for all SP $\in$ ASPEC, Catmod(SP) is a subcategory of 
Catmod(Sig(SP). Then we have obtained a logical framework for our approach where SPEC $=$ ASPEC Mod $=$ Catmod and Alg = Catmod restricted to SIGN. For instance, the equational algebraic specification logic where SIGN is the category of algebraic signatures (considered as specifications with no axioms) provides such a framework. If we consider behavioural specifications in the sense of [Orejas et al. 89] then amalgamations only exist if their corresponding pushouts have the "observation preserving property" (cf. [Orejas et al. 89]). Hence, in this case, our constructions will work only if this condition is satisfied.

Note also that the first order specification logic (with arbitrary first order axioms) provides a suitable framework for our approach if SIGN is the category of first order signatures.

\section{System Specifications and Modules: The Stratified Loose Approach Revisited}

Based on the general logical framework described in the last section we will develop a theory for the modular construction of implementations of modular system specifications. As a main objective of our approach we will provide a semantical basis which allows the separate implementation of the single modules of a system specification such that the local correctness of each "implementation piece" automatically implies the global correctness of the whole system implementation (principle of modular implementation).

In order to provide the motivation and the formal background of our general concept we consider in this section the particular case where a modular system specification is constructed by a hierarchy of enrichments. Our presentation follows the ideas of the stratified loose approach to the semantics of modular system specifications as proposed by [Bidoit 87]. Any enrichment step is denoted by a specification module of the form $M=(S Y S, S P)$ where SYS is a modular (sub-)system specification and SP is a specification in SPEC (cf. Section 2), called module body, which defines the properties of the enrichment. Since we do not assume anything on the particular form of specifications in SPEC the module body must be a specification and cannot be a specification fragment. However, for particular instantiations of SPEC, a module body could also be defined by some explicite enrichment $\triangle S P$. For instance, in PLUSS (cf. [Bidoit 89]) and in ASL (cf. [Wirsing 86]) an explicite enrichment $\Delta$ SP would consist of some sorts, operations and axioms. It is necessary to remark that any specification in SPEC defines a system specification but not vice versa. The difference will be pointed out in the following when developing our syntactical and semantical basis for modular system design.

According to the modularity principle described above an implementation of a system specification should be constructed by separate implementations of each enrichment step, i.e. each module used for the construction of the system specification should be implementable independently from each other. Hence it is necessary to strongly distinguish on the syntactical and on the semantical level between a module $\mathrm{M}=$ (SYS, SP) defining an enrichment of SYS and the system specification Sys(M) resulting from the enrichment. In order to start the process of constructing system specifications by successive enrichments of already given system specifications we need an empty system specification, denoted by Emp. Then the (abstract) syntax of modules and their associated system specifications is defined as follows: (Note that the syntax of system specifications will be generalized in definition 5 and definition 7 
in order to take into account module application.)

\section{Definition 1}

(0) $E m p$ is a system specification with signature $\operatorname{Sig}(\mathrm{Emp}){ }_{\text {def }} \emptyset$. Emp is called the empty system specification.

(1) If SYS is a system specification and SP is a specification in SPEC such that $\operatorname{Sig}(\mathrm{SYS}) \subseteq \operatorname{Sig}(\mathrm{SP})$, then

$$
\mathrm{M}=(\mathrm{SYS}, \mathrm{SP})
$$

is a specification module (also called module for short) with signature $\operatorname{Sig}(\mathrm{M})={ }_{\text {def }}$ $\operatorname{Sig}(\mathrm{SP})$.

(2) If $M=(S Y S, S P)$ is a specification module, then

$$
\text { Sys(M) }
$$

is a system specification with signature $\operatorname{Sig}(\operatorname{Sys}(M))={ }_{\operatorname{def}} \operatorname{Sig}(M)$. Sys(M) is called the "resulting system specification obtained by applying the enrichment $M$ to SYS".

Notation: Any specification SP in SPEC defines an associated system specification $\operatorname{Sys}(\mathrm{SP})=_{\text {def }} \operatorname{Sys}(\mathrm{M})$ where $\mathrm{M}=(\mathrm{Emp}, \mathrm{SP})$.

As a consequence of the above definition system specifications are of the form Sys(...Sys(Sys(Sys(SP1), SP2), SP3)..., SPn) where SP1, .., SPn are specifications in SPEC. A more general construction of system specifications will be defined in the next sections. Note that if SPEC allows to define reachability constraints (as e.g. in PLUSS or ASL) then system specifications provide a flexible setting describing data structures with constraints.

According to the loose approach, the semantics of a modular system specification SYS will be defined as some class (MSYS) of Sig(SYS)-algebras, called the model class of SYS. Given some realization (program) P, its correctness w.r.t. a system specification SYS can then be established by relating the program $P$ to one of the models of SYS. In order to study the impact of modularity on the semantics of modules and system specifications we will, for the moment, adopt a simplified view where implementations are treated as models of a specification. A more general framework of implementations will be developed in the next section.

In order to define the semantics of modules and system specifications we first summarize our basic requirements w.r.t. the modular development of modular software systems:

Requirements for modular system development:

1. The various specification modules of a system specification should be implementable independently of each other, possibly by different programmer teams. This means that it should be possible to implement a module $M=(S Y S, S P)$ without knowing which specific realization of the sub-system specification SYS has been (or will be) chosen. Hence, replacing some correct realization, say P1, of the system specification SYS by another correct realization of SYS, say P2, should still produce a correct realization of the whole system, without modification of the realization of the module $\mathrm{M}$.

2. Any correct realization of a system specification should also provide a correct realization of each sub-system specification (if it is restricted to the signature of the 
sub-system).

3. Any piece of software $P$ that is a correct realization of a sub-system should be reusable (as it is) for a realization of an overall system specification, i.e. no recoding of $P$ should be performed.

4. Global system implementations should be constructed in a modular way using realizations of the single modules.

According to the first requirement a realization of a module $\mathrm{M}=$ (SYS, SP) is considered to be a functor F mapping any realization A of SYS to a model of SP1). Hence, once a realization of $M$ is provided, it will produce an algebra with the desired properties independently from the particular choice of the realization of SYS. But note that this is not sufficient for satisfying our second requirement from above since the restriction $\mathrm{U}(\mathrm{F}(\mathrm{A})$ ) w.r.t. the forgetful functor $\mathrm{U}$ (mapping $\mathrm{Sig}(\mathrm{SP})$-algebras to Sig(SYS)-algebras) may not be a model of SYS. But even if we require $U(F(A)) \in$ $\mathcal{M}(S Y S)$ for all $A \in \mathcal{M}(S Y S)$ the third requirement could be violated if $U(F(A)) \neq$ $A$ because in this case the functor $F$ has modified the realization A of SYS. These considerations lead to the definition of module semantics as given below.

Now, taking into account the fourth requirement, there is only one canonical way to define the semantics of a system specification Sys(M) with $M=($ SYS, SP): The semantics of Sys(M) is defined to be the class of all $\operatorname{Sig}(\mathrm{SP})$-algebras $F(A)$ which are obtained by application of some realization $\mathrm{F}$ of $\mathrm{M}$ to some model A of SYS. Hence all realizations of the system specification $\operatorname{Sys}(\mathrm{M})$ are constructed using realizations of $M$ and of SYS. As already indicated above the semantics of a system specification SYS will be denoted by $\mathcal{M}(\mathrm{SYS})$. For the semantics of a module $M$ we use the notation SEM(M).

\section{Definition 2}

(0) Let $E m p$ be the empty system specification. Then $C M(E m p)={ }_{\text {def }} 1$ where 1 denotes the category which contains only one object.

(1) Let $\mathrm{M}=(\mathrm{SYS}, \mathrm{SP})$ be a specification module. Then $\mathrm{SEM}(\mathrm{M})={ }_{\operatorname{def}}\{\mathrm{F}: \mathcal{M}(\mathrm{SYS}) \rightarrow \operatorname{Alg}(\operatorname{Sig}(\mathrm{SP})) \mid \mathrm{F}$ is a functor such that for all $A \in M$ MYS $): F(A) \in \operatorname{Mod}(S P)$ and $U(F(A))=A$, where $U$ is the forgetful functor .

The functors of SEM(M) are called functorial models of $\mathrm{M}^{1)}$

(2) Let Sys(M) be a system specification associated to a module $M=(S Y S, S P)$. Then $\mathcal{M}(\operatorname{Sys}(\mathrm{M}))=_{\operatorname{def}}\{\mathrm{F}(\mathrm{A}) \mid \mathrm{F} \in \mathrm{SEM}(\mathrm{M}), \mathrm{A} \in \mathcal{M}(\mathrm{SYS})\}$

System specifications and specification modules are called correct if they have at least one realization:

Definition 3 A system specification SYS is correct if and only if $\mathcal{M}$ (SYS) $\neq \varnothing$. A specification module $\mathrm{M}$ is correct if and only if $\operatorname{SEM}(\mathrm{M}) \neq \emptyset$.

From the semantic definitions follows that for any specification SP in SPEC the semantics of the associated system specification Sys(SP) is the model class of SP, i.e. $\mathcal{C M}(\operatorname{Sys}(\mathrm{SP}))=\operatorname{Mod}(\mathrm{SP})$. More generally, the semantics of a system specification Sys(...Sys(Sys(Sys(SP1), SP2), SP3)..., SPn) with specifications SP1, .., SPn in SPEC is included in the semantics of SPn, i.e. CM(Sys(...Sys(Sys(Sys(SP1), SP2), SP3)..., SPn)) $\subseteq$ Mod(SPn). The following simple example shows that this inclusion

\footnotetext{
1) Note that an alternative would be to consider only functions instead of functors, as discussed e.g. in [Gaudel 92].
} 
may be a proper one. Intuitively the reason for this fact is that the semantics of system specifications takes into account our needs w.r.t. modular software development while the semantics of specifications is mainly property oriented, i.e. it accepts all algebras which satisfy the properties of a specification.

Example Consider the logical framework of equational algebraic specifications (with loose semantics) and two specifications SP1 and SP2 with exactly one sort S and two constants $a, b: \rightarrow s$. Let SP2 have the axiom $a=b$ while $\mathrm{SP} 1$ has no axiom. Then the module $\mathrm{M}=(\operatorname{Sys}(\mathrm{SP} 1), \mathrm{SP} 2)$ has no realization since, obviously, no functor $\mathrm{F}$ : $\mathcal{M}(\operatorname{Sys}(\mathrm{SP} 1)) \rightarrow \operatorname{Mod}(\mathrm{SP} 2)$ exists with $\mathrm{U}(\mathrm{F}(\mathrm{A}))=\mathrm{A}$ for all $\mathrm{A} \in \mathcal{M}(\operatorname{Sys}(\mathrm{SP} 1))=$ $\operatorname{Mod}(\mathrm{SP} 1)$. Hence no realization of Sys(M) can be produced, i.e. Sys(M) has no model. On the other hand SP2 has all algebras where a, b are interpreted as the same object as models.

\section{Implementation of Specification Modules and System Specifications}

In the last section we have essentially recalled the basic motivations and definitions of the stratified loose semantics approach introduced in [Bidoit 87]. A methodologically important objective which was not addressed there concerns the stepwise construction of implementations. After splitting an implementation task into several subtasks (according to the modular structure of a system specification) it should be possible to implement each subtask stepwise by a series of design decisions. The principle of stepwise refinement allows to separate implementation constructions into encapsulated implementation units thus leading to a programming discipline which increases the reliability of a final software product. Moreover, it provides means to document single design decision thus facilitating the maintenance of software systems.

In order to allow stepwise refinements in the stratified loose approach we cannot keep the simplified view of the last section where implementations were considered as models of a specification (or functors of a module). We have to provide means which allow to restrict successively the class of admissible realizations of modules and system specifications according to particular design decisions, like choice of algorithms, choice of data structures etc.. As a consequence, an implementation of a system specification must be a system specification as well which just has less models than its corresponding abstract specification. Analogously, an implementation of a specification module $M$ is a module as well which specifies less functors than $M$. In particular, this implies that the functors of an implementing module have the same domain as the functors of the abstract module. Hence we require that concrete and abstract modules are built on the same sub-system specification. (Note that one could even allow that the sub-system specification SYS' of an implementing module M' has more realizations than the sub-system specification SYS of the corresponding abstract module $M$ since it is sufficient that all functorial models of $M^{\prime}$ accept all realizations of SYS. For sake of simplicity we do not consider this more general condition here.)

Definition 4 (1) Let SYS and SYS' be system specifications such that Sig(SYS) = Sig(SYS'). Then SYS' is called system implementation of SYS (written SYS > SYS') if (MSYS') $\subseteq \mathcal{M}\left(\mathrm{SYS}^{\prime}\right.$ ).

(2) Let $M=(S Y S, S P)$ and $M^{\prime}=(S Y S, S P ')$ be specification modules such that $\operatorname{Sig}(M)=\operatorname{Sig}\left(M^{\prime}\right)$. Then $M^{\prime}$ is called module implementation of $M$ (written $M$ $\left.\sim \sim \sim>_{\bmod } M^{\prime}\right)$ if SEM(M') $\subseteq \operatorname{SEM}(M)$. 
In the above implementation definitions it is required that the signatures of concrete and abstract specifications (modules resp.) coincide. A more general concept which allows to define implementations relatively to a signature morphism from an abstract signature to a concrete one will be considered in the next section.

The power of our implementation mechanism depends on the underlying specification language SPEC. For instance, if SPEC incorporates features for behavioural abstractions (like in ASL or in [Sannella, Tarlecki 87]) or if it is directly equipped with behavioural semantics (cf. e.g. [Bernot, Bidoit 91], [Hennicker 92]) then the implementation definition reflects the intuitive idea that a realization is correct if it satisfies the desired input/output behaviour.

The following fact is an immediate consequence of the definitions. It says that module implementations induce system implementations for their associated systems:

Fact 1 Let $M=(S Y S, S P)$ and $M^{\prime}=(S Y S, S P ')$ be modules such that $M \sim \sim$ mod $M^{\prime}$. Then Sys $(M) \sim \sim>$ sys $\operatorname{Sys}\left(M^{\prime}\right)$.

We will now analyze how a modular implementation of a system specification will be constructed with the help of our implementation mechanism. Since a system specification is built up by a sequence of modules, each single module enriching a given sub-system specification, it should be enough to implement each module in order to obtain a global system implementation. According to the inductive construction of system specifications this problem can be reduced to the following case: Let SYS1 be a system specification which is constructed by applying a module $\mathrm{M}=(\mathrm{SYS}, \mathrm{SP})$ to a sub-system specification SYS, i.e. SYS1 = Sys(M). Assume that a system implementation SYS' of the subsystem SYS is already given. The question is how to construct a system implementation for SYS1 using SYS' and a given module implementation $M \sim \sim>$ mod $M^{\prime}$ where $M^{\prime}=$ (SYS, SP'). Although, according to the above fact, Sys(M') provides a system implementation of SYS1 this is not the implementation we want since it does not use SYS'.

As a solution we propose to introduce a new operation on modules which allows to instantiate sub-system specifications by sub-system implementations. The result of the application of the module $M^{\prime}=($ SYS, SP') to the implementation SYS' of SYS yields a system specification which contains as models all algebras which can be produced by the application of functorial models of M' to realizations of SYS'. Before giving the formal definition of the application we will discuss another attempt for solving the problem of sub-system implementation which, however, is not successful:

Remark: Considering the above situation one could try to textually replace within the module $M$ ' the sub-system specification SYS by its implementation SYS' thus yielding a module $\mathrm{M}_{\text {repl }}=\left(\mathrm{SYS}^{\prime}, \mathrm{SP}^{\prime}\right)$. But then it is not guaranteed that $\operatorname{Sys}\left(\mathrm{M}_{\text {repl }}\right)$ is a system implementation of SYS1. The reason is that, since $\mathcal{M}\left(\mathrm{SYS}^{\prime}\right) \subseteq \mathcal{M}$ (SYS), there might exist functorial models $\mathrm{F}_{\text {repl }}: \mathcal{M}$ (SYS') $\rightarrow \operatorname{Alg}\left(\operatorname{Sig}\left(\mathrm{SP}^{\prime}\right)\right)$ of $\mathrm{M}_{\text {repl }}$ which

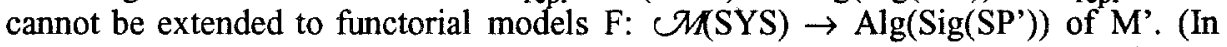
other words $F_{\text {repl }}$ may not be a restriction of some functorial model $F$ of $M^{\prime}$.) Consequently, such functors might produce algebras which cannot be produced by functorial models of $\mathrm{M}^{\prime}$ and hence do not belong to the models of the system specification SYS1 to be implemented. 


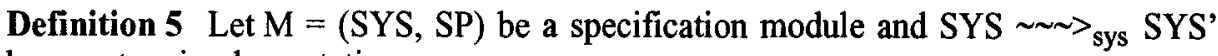
be a system implementation.

Then $\mathrm{M}\left[\mathrm{SYS}=>\mathrm{SYS}^{\prime}\right]$ is a system specification, called the application of $M$ to $S Y S^{\prime}$, with signature $\operatorname{Sig}\left(\mathrm{M}\left[\mathrm{SYS} \Longrightarrow\right.\right.$ SYS']) $=_{\operatorname{def}} \operatorname{Sig}(\mathrm{SP})$ and with semantics $\mathcal{M}\left(\mathrm{M}^{\prime}\left[\mathrm{SYS} \Rightarrow \mathrm{SYS}^{\prime}\right]\right)=_{\operatorname{def}}\left\{\mathrm{F}(\mathrm{A}) \mid \mathrm{F} \in \mathrm{SEM}(\mathrm{M}), \mathrm{A} \in \mathcal{M}\left(\mathrm{SYS}^{\prime}\right)\right\}$

Note that $\mathcal{M}(\mathrm{M}[\mathrm{SYS} \Longrightarrow \mathrm{SYS}])=\mathcal{M}$ Sys(M)), i.e. the system specification associated to a module $M$ has the same semantics as the application of $M$ to its own sub-system specification SYS.

The following lemma states some important properties of module application which can be easily derived from the definitions:

Lemma 2 Let $M=(S Y S, S P), M^{\prime}=(S Y S, S P ')$ be modules with $M \sim \sim>_{\text {mod }} M^{\prime}$ and let SYS and SYS' be system specifications such that SYS $\sim \sim>_{\text {sys }}$ SYS'. Then the following holds:

(1) $\mathrm{M}\left[\mathrm{SYS}=\Rightarrow \mathrm{SYS}^{\prime}\right]$ is a correct system specification if and only if $\mathrm{M}$ is a correct specification module and SYS' is a correct system specification.

(2) $\operatorname{Sys}(\mathrm{M}) \sim \sim \sim>_{\text {sys }} \mathrm{M}^{\prime}\left[\mathrm{SYS}=\mathrm{SYS}^{\prime}\right]$

(3) If $\mathrm{M}^{\prime}$ is correct then $\mathcal{M}\left(\mathrm{SYS}^{\prime}\right)=\mathrm{U}\left(\mathcal{M}\left(\mathrm{M}^{\prime}[\mathrm{SYS}=\Rightarrow\right.\right.$ SYS'])) where $\mathrm{U}$ : $\mathrm{Alg}\left(\operatorname{Sig}\left(\mathrm{M}^{\prime}\right)\right) \rightarrow \mathrm{Alg}\left(\operatorname{Sig}\left(\mathrm{SYS}^{\prime}\right)\right)$ is the forgetful functor.

We shall give a short interpretation of the above properties:

Property (1) shows that the application of a correct module to a correct system specification yields again a correct system specification. In connection with property (2) this means that "locally" correct implementations can be composed to a "globally" correct implementation of a system specification. In particular, property (2) says that implementations can be developed independently from each other, i.e. an implementation of the module $\mathrm{M}$ can be constructed without knowing which implementation of the sub-system specification SYS will be provided and vice versa. This is exactly what we have postulated in our first requirement for modular system design in Section 3.

Property (3) ensures that the application of a module implementation $M^{\prime}$ to a subsystem implementation SYS' does not change (the semantics of) the sub-system implementation. Hence sub-system implementations will be reused without modification. In particular, the application of $M^{\prime}$ to SYS' yields also an implementation of the smaller system SYS' if it is restricted to the signature of SYS'. This is exactly what we have postulated in the second and third requirement for modular system design in Section 3. Note that the application of a module to a system specification corresponds to the application of a functor to an algebra. Having this correspondence in mind we see that property (3) corresponds at the specification level exactly to the semantical functor property $" \mathrm{U}(\mathrm{F}(\mathrm{A}))=\mathrm{A}$ " required for the functorial models $\mathrm{F}$ of a module.

\section{A Uniform Concept for Implementation and Parameterization}

In the previous section we have assumed that the signatures of implementations and abstract system specifications (modules resp.) coincide. We will now drop this assumption thus leading to a generalized implementation concept which turns out to capture our ideas on the parameterization of system specifications as well.

In practice implementations are usually built on top of already existing, standard data 
structures. Hence the connection between an abstract specification and the implementation has usually to be provided by a signature morphism which maps the abstract types (sorts) and operations to concrete ones. In order to incorporate this idea in our approach we extend the implementation definitions given in the last section as follows:

Definition 6 (1) Let SYS and SYS' be system specifications and $\sigma: \operatorname{Sig}(S Y S) \rightarrow$ $\operatorname{Sig}\left(\mathrm{SYS}^{\prime}\right)$ be a signature morphism. Then SYS' is called $\sigma$-system implementation of SYS (written SYS $\sigma^{\sim \sim>}$ sys $\left.S Y S^{\prime}\right)$ if $\left.U_{\sigma}\left(\mathcal{M M S Y S}^{\prime}\right)\right) \subseteq \mathcal{M}(\mathrm{SYS})$.

(2) Let $M=(S Y S, S P)$ and $M^{\prime}=(S Y S, S P ')$ be specification modules and $\tau$ : $\operatorname{Sig}(M)$ $\rightarrow \operatorname{Sig}\left(\mathrm{M}^{\prime}\right)$ be a signature morphism. Then $\mathrm{M}^{\prime}$ is called $\tau$-module implementation of $\mathrm{M}$ (written $\left.\mathrm{M}_{\tau \sim \sim \sim} \sim \mathrm{P}_{\bmod } \mathrm{M}^{\prime}\right)$ if $\mathrm{U}_{\tau}\left(\operatorname{SEM}\left(\mathrm{M}^{\prime}\right)\right) \subseteq \operatorname{SEM}(\mathrm{M})$ where

$$
\mathrm{U}_{\tau}\left(\mathrm{SEM}\left(\mathrm{M}^{\prime}\right)\right)=_{\operatorname{def}}\left\{\mathrm{U}_{\tau} \bullet \mathrm{F}^{\prime} \mid \mathrm{F}^{\prime} \in \operatorname{SEM}\left(\mathrm{M}^{\prime}\right)\right\}
$$

Fact 2 If SYS $\sigma \sim \sim>_{\text {sys }}$ SYS' and SYS' $\sigma^{\circ \sim \sim>\text { sys }}$ SYS" then SYS $\sigma \bullet \sigma \sim \sim>$ sys

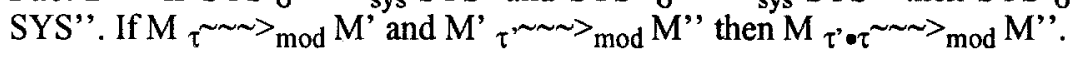

We can now extend the definition of module application to the case of $\sigma$-system implementations:

Definition 7 Let $\mathrm{M}=(\mathrm{SYS}, \mathrm{SP})$ be a specification module and SYS $\sigma^{\sim \sim>}$ sys $S Y S$ ' be a $\sigma$-system implementation.

Then $\mathrm{M}[\mathrm{SYS}=\sigma \Rightarrow$ SYS'] is a system specification, called the application of $M$ to SYS' w.r.t. $\sigma$.

The signature $\operatorname{Sig}\left(\mathrm{M}\left[\mathrm{SYS}=\sigma \Rightarrow \mathrm{SYS}^{\prime}\right]\right)={ }_{\text {def }} \mathrm{PO}(1, \sigma)$ is the pushout of the following diagram in the category SIGN of signatures where $\imath$ denotes the inclusion morphism:

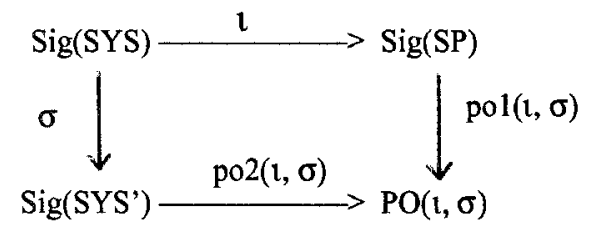

The semantics of the module application is defined by

$\mathcal{M}\left(\mathrm{M}\left[\mathrm{SYS}=\sigma \Rightarrow \mathrm{SYS}^{\prime}\right]\right)=_{\operatorname{def}}\left(\mathrm{F}^{\prime}\left(\mathrm{A}^{\prime}\right) \mid \mathrm{A}^{\prime} \in \mathcal{M}\left(\mathrm{SYS}^{\prime}\right)\right.$ and $\mathrm{F}^{\prime}: \mathcal{M}\left(\mathrm{SYS}^{\prime}\right) \rightarrow$ $\operatorname{Alg}(\operatorname{PO}(l, \sigma))$ is the unique extension of some $F \in \operatorname{SEM}(M)$ w.r.t. $\sigma\}$

By lemma 1, the unique extension $F^{\prime}$ of $F$ in the above definition exists since $\mathrm{U}_{\sigma}\left(\mathcal{M}\left(\mathrm{SYS}^{\prime}\right)\right) \subseteq \mathcal{M}\left(\mathrm{SYS}^{\prime}\right)$ and since for all functorial models $F \in \operatorname{SEM}(M)$, $\mathrm{U}(\mathrm{F}(\mathrm{A}))=\mathrm{A}$ holds (where $\mathrm{U}=\mathrm{U}_{1}$ ). Moreover, by lemma 1 , the semantics of the application of the module $M$ to a system specification SYS' can be characterized by all amalgamations which can be constructed by the models of SYS' and by the functorial models of $\mathrm{M}$, i.e. we have the following fact:

Fact $3 \mathscr{M}(\mathrm{M}[\mathrm{SYS}=\sigma \Rightarrow \mathrm{SYS}])=\left\{\mathrm{A} \in \mathrm{A} \lg (\mathrm{PO}(\mathrm{l}, \sigma)) \mid \mathrm{A}=\mathrm{A}^{\prime} \oplus \mathrm{F}\left(\mathrm{U}_{\sigma}\left(\mathrm{A}^{\prime}\right)\right)\right.$ where $A^{\prime} \in \mathcal{M}\left(S_{Y} S^{\prime}\right)$ and $\left.F \in \operatorname{SEM}(M)\right\}$

Note that the result signature of the application is not $\operatorname{Sig}(\operatorname{SP})$ but $\mathrm{PO}(1, \sigma)$ since the instantiation of the subsystem SYS by an implementation SYS' should produce an implementation of the overall system specification $\operatorname{Sys}(M)$ via a signature morphism 
that preserves (i.e. extends) the signature morphism $\sigma$ used for the implementation of the subsystem SYS. Using our constructions one can show that M[SYS $\left.=\sigma \Rightarrow S Y S^{\prime}\right]$ is indeed an implementation of $\operatorname{Sys}(\mathrm{M})$ via the pushout morphism po1 $(1, \sigma)$ which extends $\sigma$ to $\operatorname{Sig}(\mathrm{SP})$. (The proof is a particular consequence of theorem 1 below using the property $\mathcal{M}(\operatorname{Sys}(\mathrm{M}))=\mathcal{M}(\mathrm{M}[\mathrm{SYS}=\mathrm{id}=>\mathrm{SYS}])$ where id denotes the identity morphism.)

Notation: In order to avoid syntactical overhead we will also write M[SYS'] instead of $\mathrm{M}[\mathrm{SYS}=\sigma \Rightarrow$ SYS'] if the explicite denotation of SYS and $\sigma$ is not important or clear from the context.

An obvious, but important property of the application operation is that it preserves the correctness of the single constituent parts of a system specification:

Fact $4 \mathrm{M}\left[\mathrm{SYS}=\sigma \Rightarrow \mathrm{SYS}^{\prime}\right]$ is a correct system specification if and only if $M$ is a correct specification module and SYS' is a correct system specification.

The above formalism allows to apply a module to an implementation of its sub-system specification. Since implementations are specializations which, on the one hand, may add "something" (usually some sorts and operations) to the signature of the abstract specification and, on the other hand, may rename some parts of the abstract signature, there is, due to the loose semantics approach, conceptually no difference between implementation and parameter actualization (in the classical sense of parameterized specifications). Hence our general framework can be interpreted in two ways:

First, a module may be seen to define an extension of a sub-system specification. Then the application operation is used for instantiating the sub-system specification by an implementation. Secondly, a module $M=$ (SYS, SP) may be interpreted as a parameterized specification with formal parameter specification SYS. In this case, the application of a module is viewed as actualization (or parameter passing). Instead of sub-system implementations we then speak of actual parameters. For instance, the following specification module STACK-MOD is supposed to specify stacks over arbitrary elements. The formal parameter is defined by a specification ELEM for the elements and the stack operations are specified in the specification STACK:

STACK-MOD $=($ Sys(ELEM $)$, STACK $)$.

Then, if INT is a specification of integers, stacks of integers can be defined by the following actualization: STACK-MOD[Sys(INT)].

The actualization is performed w.r.t. an appropriate signature morphism which is not explicitely denoted here. Due to the pushout construction no name conflicts will occur in the actualization.

In the following we will neither concentrate only on the implementation aspect nor only on the parameterization aspect of our approach but we will discuss the various correlations between both.

As a first example, let $\mathrm{M}=(\mathrm{SYS}, \mathrm{SP})$ be a specification module where SYS is considered as a formal parameter specification. Then it is an important question whether applications of $M$ to actual parameters preserve implementation relations between the actual parameters. This property is well known as monotonicity of parameterized specifications. For instance, if Sys(INT-IMPL) is an implementation of Sys(INT) (e.g. implementing integers by bit strings) then STACK-MOD[Sys(INTIMPL)] should be automatically an implementation of STACK-MOD[Sys(INT)]. The 
following theorem states that this is indeed true for arbitrary modules and arbitrary implementation relations for actual parameters:

\section{Theorem 1 (Monotonicity)}

Let $M=(S Y S, S P)$ be a specification module and let SYS, SYS' and SYS' be system specifications such that SYS $\sigma^{\sim \sim>}$ sys SYS' (where SYS' is seen as an actual parameter) and SYS' $\sigma^{\sim \sim \sim>}$ sys $S Y S$ " (where SYS" is seen as an implementation of the actual parameter).

Then M[SYS $=\sigma=>$ SYS'] $\kappa^{\sim \sim>}$ sys $M\left[S Y S=\sigma^{\prime} \bullet \sigma \Rightarrow>\right.$ SYS'] is a system implementation where $\kappa$ is the unique signature morphism determined by the following pushout diagrams:

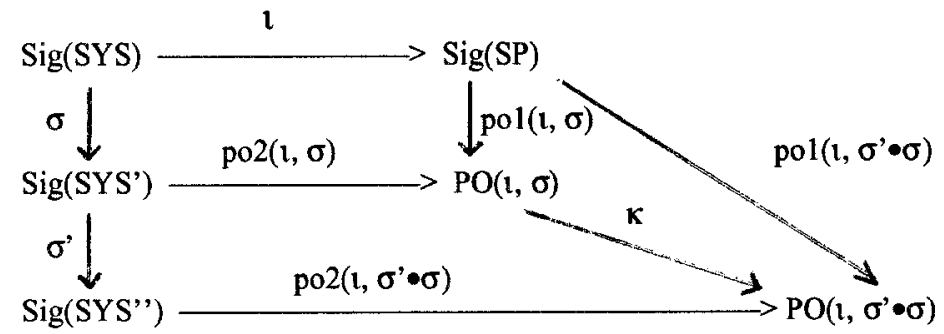

Proof: First, note that M[SYS $=\sigma^{\prime} \bullet \sigma \Rightarrow$ SYS'] is well defined since system implementation is transitive (cf. fact 2). We have to show that $\mathrm{U}_{\kappa}(\mathcal{C M} \mathrm{M}$ [SYS $=\sigma^{\prime} \bullet \sigma \Rightarrow$ SYS'] $\left.)\right) \subseteq(M(M[S Y S=\sigma \Rightarrow$ SYS']). By fact 3 , $\mathcal{M}\left(\mathrm{M}\left[\mathrm{SYS}=\sigma^{\prime} \bullet \sigma=>\mathrm{SYS}^{\prime \prime}\right]\right)=\left\{\mathrm{A} \in \operatorname{Alg}\left(\mathrm{PO}\left(1, \sigma^{\prime} \bullet \sigma\right)\right) \mid \mathrm{A}=\mathrm{A}{ }^{\prime \prime} \oplus \mathrm{F}\left(\mathrm{U}_{\sigma^{\prime} \bullet \sigma}\left(\mathrm{A}^{\prime \prime}\right)\right)\right.$ where A" $\in$ (MSYS") and $F \in \operatorname{SEM}(M)\}$ and $\left.\mathcal{M M}\left[\mathrm{SYSS}^{\prime} \sigma \mathrm{SYS}^{\prime}\right]\right)=\left\{\mathrm{A} \in \operatorname{Alg}(\mathrm{PO}(\mathrm{l}, \sigma)) \mid \mathrm{A}=\mathrm{A}^{\prime} \oplus \mathrm{F}\left(\mathrm{U}_{\sigma}\left(\mathrm{A}^{\prime}\right)\right)\right.$ where $\mathrm{A}^{\prime} \in$ $\mathcal{M}$ (SYS') and $\mathrm{F} \in \mathrm{SEM}(\mathrm{M})\}$.

Now, let $A^{\prime \prime} \oplus \mathrm{F}\left(\mathrm{U}_{\sigma \cdot \bullet \sigma}\left(\mathrm{A}^{\prime \prime}\right)\right) \in \mathcal{M} \mathrm{M}\left[\mathrm{SYS}=\sigma^{\prime} \bullet \sigma \Rightarrow \mathrm{SYS}\right.$ '] $\left.]\right)$ with $\mathrm{A}^{\prime \prime} \in$ $\mathcal{M}$ (SYS") and $\mathrm{F} \in \mathrm{SEM}(\mathrm{M})$.

Then, by the commutativity of the diagrams and by the properties of amalgamated unions, $\mathrm{U}_{\mathrm{po2}(\mathrm{h}, \sigma)}\left(\mathrm{U}_{\mathrm{K}}\left(\mathrm{A}^{\prime \prime} \oplus \mathrm{F}\left(\mathrm{U}_{\sigma^{\prime} \bullet \sigma}\left(\mathrm{A}^{\prime \prime}\right)\right)\right)\right)=\mathrm{U}_{\sigma},\left(\mathrm{U}_{\mathrm{p} 02\left(\left\llcorner, \sigma^{\prime} \bullet \sigma\right)\right.}\left(\mathrm{A}^{\prime \prime} \oplus \mathrm{F}\left(\mathrm{U}_{\sigma \cdot \bullet \sigma}\left(\mathrm{A}^{\prime \prime}\right)\right)\right)\right)$ $=\mathrm{U}_{\sigma^{\prime}}\left(\mathrm{A}^{\prime \prime}\right)$ and

$\mathrm{U}_{\mathrm{pol}(1, \sigma)}\left(\mathrm{U}_{\mathrm{k}}\left(\mathrm{A}^{\prime \prime} \oplus \mathrm{F}\left(\mathrm{U}_{\sigma \cdot \bullet \sigma}\left(\mathrm{A}^{\prime \prime}\right)\right)\right)\right)=\mathrm{U}_{\mathrm{pol}(\mathrm{l}, \sigma \bullet \sigma)}\left(\mathrm{A}^{\prime \prime} \oplus \mathrm{F}\left(\mathrm{U}_{\sigma \cdot \bullet \sigma}\left(\mathrm{A}^{\prime \prime}\right)\right)\right)=$ $\mathrm{F}\left(\mathrm{U}_{\sigma}\left(\mathrm{U}_{\sigma},\left(\mathrm{A}^{\prime \prime}\right)\right)\right)$. Hence, by the uniqueness of amalgamations, we know that $\mathrm{U}_{\mathrm{K}}\left(\mathrm{A}^{\prime \prime}\right.$ $\left.\oplus F\left(U_{\sigma} \cdot \circ\left(A^{\prime \prime}\right)\right)\right)=U_{\sigma},\left(A^{\prime \prime}\right) \oplus F\left(U_{\sigma}\left(U_{\sigma^{\prime}}\left(A^{\prime \prime}\right)\right)\right)$. Since $U_{\sigma^{\prime}}\left(A^{\prime \prime}\right) \in M$ MSYS') and $F$ $\in \operatorname{SEM}(M)$ we then know (by fact 3$)$ that $U_{\mathcal{K}}\left(A^{\prime \prime} \oplus \mathrm{F}\left(\mathrm{U}_{\sigma \cdot \bullet \sigma}\left(\mathrm{A}^{\prime \prime}\right)\right)\right) \in \mathcal{M}(\mathrm{M}[\mathrm{SYS}$ $=\sigma=>$ SYS'] $^{\prime}$ and we are done.

We will now consider the relationship between module implementations and parameter passing. An important question is whether module implementations induce automatically system implementations for arbitrary actualizations, i.e. whether parameter passing is compatible with module implementation. For instance, given a module implementation STACK-MOD-IMPL $=($ Sys(ELEM), STACK-IMPL) of the module STACK-MOD (e.g. implementing stacks by arrays with pointers) and an arbitrary actual parameter, e.g. Sys(INT), of both modules, then we would like that the actualization STACK-MOD-IMPL[Sys(INT)] yields automatically a system implementation of the actualization STACK-MOD[Sys(INT)]. The next theorem states the compatibility of module implementation with parameter passing: 
Theorem 2 (Compatibility of module implementation with parameter passing) Let $\mathrm{M}=(\mathrm{SYS}, \mathrm{SP}), \mathrm{M}^{\prime}=\left(\mathrm{SYS}, \mathrm{SP}^{\prime}\right)$ be specification modules such that $\mathrm{M}_{\tau \sim \sim>}$ mod $M^{\prime}$ and let SYS' be a system specification such that $S Y S_{\sigma} \sim \sim>$ sys $S Y S$ '.

Then $\mathrm{M}\left[\mathrm{SYS}=\sigma \Rightarrow \mathrm{SYS}^{\prime}\right]_{\mu} \sim \sim>>_{\text {sys }} \mathrm{M}^{\prime}\left[\mathrm{SYS}=\sigma \Rightarrow \mathrm{SYS}^{\prime}\right]$ is a system implementation where $\mu$ is the unique signature morphism determined by the following pushout diagrams:

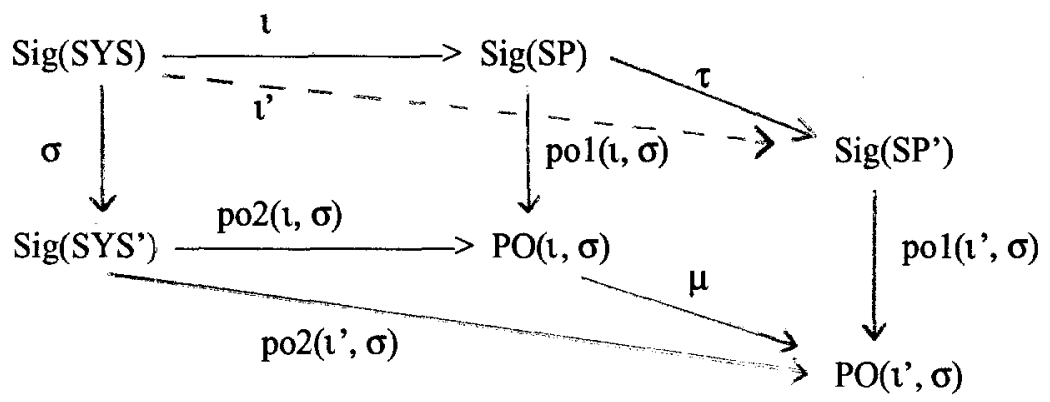

Proof: We have to show that $\left.\mathrm{U}_{\mu}\left(\mathcal{M} \mathrm{M}^{\prime}[\mathrm{SYS}=\sigma \Rightarrow \mathrm{SYS}]\right)\right) \subseteq \mathcal{M}$ M[SYS $=\sigma=>$ SYS']). By fact 3,

$\mathcal{M}\left(\mathrm{M}^{\prime}\left[\mathrm{SYS}=\sigma \Rightarrow \mathrm{SYS}^{\prime}\right]\right)=\left\{\mathrm{A} \in \operatorname{Alg}\left(\mathrm{PO}\left(\mathrm{l}^{\prime}, \sigma\right)\right) \mid \mathrm{A}=\mathrm{A}^{\prime} \oplus \mathrm{F}^{\prime}\left(\mathrm{U}_{\sigma}\left(\mathrm{A}^{\prime}\right)\right)\right.$ where $\mathrm{A}^{\prime} \in$ $\mathcal{M}$ (SYS') and F' $\left.\in \mathrm{SEM}\left(\mathrm{M}^{\prime}\right)\right\}$ and

$\mathcal{M}\left(\mathrm{M}\left[\mathrm{SYS}=\sigma \Rightarrow \mathrm{SYS}^{\prime}\right]\right)=\left\{\mathrm{A} \in \mathrm{Alg}(\mathrm{PO}(\mathrm{l}, \sigma)) \mid \mathrm{A}=\mathrm{A}^{\prime} \oplus \mathrm{F}\left(\mathrm{U}_{\sigma}\left(\mathrm{A}^{\prime}\right)\right)\right.$ where $\mathrm{A}^{\prime} \in$ (MSYS') and F $\in$ SEM(M) $\}$.

Now, let $A^{\prime} \oplus F^{\prime}\left(U_{\sigma}\left(A^{\prime}\right)\right) \in \mathcal{M}\left(M^{\prime}\left[S Y S=\sigma \Rightarrow S Y S^{\prime}\right]\right)$ with $A^{\prime} \in \mathcal{M}\left(S Y S^{\prime}\right)$ and $F^{\prime}$ $\in \operatorname{SEM}\left(\mathrm{M}^{\prime}\right)$.

Then, by the commutativity of the lower triangle and by the properties of amalgamated unions, $U_{\text {po2 }(1, \sigma)}\left(U_{\mu}\left(A^{\prime} \oplus F^{\prime}\left(U_{\sigma}\left(A^{\prime}\right)\right)\right)\right)=U_{p 02\left(l^{\prime}, \sigma\right.}\left(A^{\prime} \oplus F^{\prime}\left(U_{\sigma}\left(A^{\prime}\right)\right)\right)=A^{\prime}$.

We will now show:

(\#) $\mathrm{U}_{\mathrm{po} 1(\mathrm{l}, \sigma)}\left(\mathrm{U}_{\mu}\left(\mathrm{A}^{\prime} \oplus \mathrm{F}^{\prime}\left(\mathrm{U}_{\sigma}\left(\mathrm{A}^{\prime}\right)\right)\right)\right)=\mathrm{F}\left(\mathrm{U}_{\sigma}\left(\mathrm{A}^{\prime}\right)\right)$ where $\mathrm{F}: \mathcal{M}(\mathrm{SYS}) \rightarrow \operatorname{Alg}(\operatorname{Sig}(\mathrm{SP}))$ is defined by $F(A)={ }_{\operatorname{def}} U_{\tau}\left(F^{\prime}(A)\right)$ for all $A \in M$ MSYS $)$, i.e. $F=U_{\tau} \bullet F^{\prime}$.

First, note that $F \in \operatorname{SEM}(M)$ since it is assumed that $M_{\tau \sim \sim>} \sim{ }_{\text {mod }} M^{\prime}$.

Now (\#) can be proved using the commutativity of the right square and the properties of the amalgamated union: $U_{\mathrm{pol}(\mathrm{l}, \sigma)}\left(\mathrm{U}_{\mu}\left(\mathrm{A}^{\prime} \oplus \mathrm{F}^{\prime}\left(\mathrm{U}_{\alpha}\left(\mathrm{A}^{\prime}\right)\right)\right)\right)=\mathrm{U}_{\tau}\left(\mathrm{U}_{\mathrm{pol}\left(\mathrm{l}^{\prime}, \sigma\right)}\left(\mathrm{A}^{\prime} \oplus\right.\right.$ $\left.\left.F^{\prime}\left(U_{\sigma}\left(A^{\prime}\right)\right)\right)\right)=U_{\tau}\left(F^{\prime}\left(U_{\alpha}\left(A^{\prime}\right)\right)\right)=F\left(U_{\sigma}\left(A^{\prime}\right)\right)$, by definition of $F$. (Note that $\mathrm{F}\left(\mathrm{U}_{\sigma}\left(\mathrm{A}^{\prime}\right)\right)$ is well defined, since SYS $\sigma^{\sim \sim \sim>}$ sys $S Y S^{\prime}$ is assumed and therefore $\mathrm{U}_{\sigma}\left(\mathrm{A}^{\prime}\right) \in \mathcal{G}(\mathrm{SYS})$.) The uniqueness of the amalgamated union now implies that $U_{\mu}\left(A^{\prime} \oplus F^{\prime}\left(U_{\sigma}\left(A^{\prime}\right)\right)\right)=A^{\prime} \oplus F\left(U_{\sigma}\left(A^{\prime}\right)\right)$. Hence, by fact $3, U_{\mu}\left(A^{\prime} \oplus F^{\prime}\left(U_{\sigma}\left(A^{\prime}\right)\right)\right)$ $\in \mathcal{C}\left(M\left[S Y S=\sigma \Rightarrow S Y S^{\prime}\right]\right)$ and we are done.

As a consequence of the above theorems we obtain the so-called horizontal composition property for module and system implementations. For instance, if STACK-MOD, STACK-MOD-IMPL, Sys(INT) and Sys(INT-IMPL) are as above, then STACK-MOD-IMPL[Sys(INT-IMPL)] yields a system implementation of STACK-MOD[Sys(INT)].

\section{Corollary 1 (Horizontal composition property)}

Let $M=(S Y S, S P), M^{\prime}=\left(S Y S, S^{\prime}\right)$ be specification modules such that $M_{\tau \sim \sim} \sim$ mod M'. Moreover, let SYS, SYS' and SYS' be system specifications such that SYS $\sigma^{\sim \sim \sim} \sim{ }_{\text {sys }}$ SYS' (where SYS' is seen as an actual parameter) and SYS' $\sigma^{\prime \sim \sim \sim>}$ sys SYS" (where SYS" is seen as an implementation of the actual parameter). 
Then we have the following diagram of system implementations:

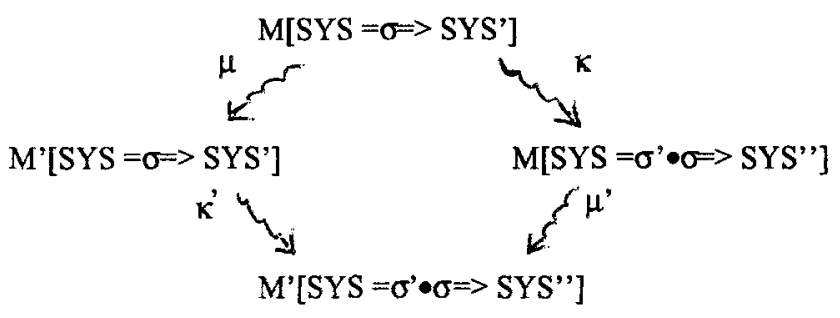

with appropriate signature morphisms $\mu, \mu^{\prime}, \kappa, \kappa^{\prime}$ as defined in the pushout diagrams of theorem 1 and theorem 2.

Proof: The implementations w.r.t. $\kappa$ and $\kappa$ ' are direct consequences of theorem 1 and the implementations w.r.t. $\mu$ and $\mu$ ' are direct consequences of theorem 2 .

Example As an example consider the following system specification SSI of sets of stacks of integers which is built by the following systems and modules:

Sys(INT) and STACK-MOD = (Sys(ELEM), STACK) are given as above,

SET-MOD $=($ Sys(ELEM), SET) has the same formal parameter as the stack module and the specification SET defines the set operations w.r.t. arbitrary elements. Then SSI is defined as SSI = SET-MOD[STACK-MOD[Sys(INT)]].

Now suppose that the following implementation modules are given (implementing e.g. stacks by arrays with pointers and sets by lists) and that Sys(INT-IMPL) is an implementation of Sys(INT):

STACK-MOD-IMPL = $($ Sys $(E L E M)$, STACK-IMPL $)$,

SET-MOD-IMPL $=($ Sys(ELEM), SET-IMPL).

Then SSI-IMPL = SET-MOD-IMPL[STACK-MOD-IMPL[Sys(INT-IMPL)] is a system implementation of SSI.

We will convince ourselves that this is true by considering the following implementation steps:

1. Since Sys(INT-IMPL) implements SYS(INT) and STACK-MOD-IMPL implements STACK-MOD, we know by the corollary that STACK-MODIMPL[Sys(INT-IMPL)] is a system implementation of STACK-MOD[Sys(INT)].

2. From 1. and from the fact that SET-MOD-IMPL is a module implementation of SET-MOD follows, again using the corollary, that SSI-IMPL is a system implementation of SSI.

Remark: A particular application of corollary 1 is the case where SYS' $=$ SYS, $\sigma=$ id (and SYS" $=$ SYS', $\left.\sigma^{\prime}=\sigma\right)$. Since $\mathcal{M}(\operatorname{Sys}(\mathrm{M}))=\mathcal{M}(\mathrm{M}[\mathrm{SYS}=\mathrm{id} \Rightarrow \mathrm{SYS}])$ we then obtain that M'[SYS $=\sigma \Rightarrow$ SYS'] is a system implementation of Sys(M). If SYS is not considered as a formal parameter but as a sub-system specification enriched by $M$, then this fact ensures the independent construction of implementations for subsystem specifications and modules. 


\section{Concluding Remarks}

We have presented a uniform framework for the construction of modular implementations which incorporates the separation of implementation tasks into smaller units and the stepwise development of single "implementation pieces". In order to achieve the independent development of implementations we have introduced two basic specification units: specification modules and system specifications. Semantically, a specification module denotes a class of (algebra-valued) functors and a system specification denotes the class of all algebras which can be constructed by (successive) functor applications according to the modular structure of the specification. Hence, the semantics of a system specification reflects the modular construction of realizations and therefore is different from ASL-like specifications. In particular this means that system specifications are different from 0th-order specifications as defined in [Sannella et al. 90]. On the other hand the semantics of a specification module corresponds to the first-order case of specifications of parametric algebras in the sense of [Sannella et al. 90] because in both concepts realizations are considered to be parameterized programs. However it is important to notice that in our approach specification modules are used as the fundamental tool for building structured (hierarchical) system specifications which makes an essential difference between specification modules and specifications of parametric algebras.

According to the loose approach we have introduced an implementation relation for modules and for system specifications and we have introduced a basic operation on modules (module application) which allows to instantiate the sub-system specification of a module by an implementation. We have discussed the close relationship between system implementation and parameterization and, in fact, we have proposed to use the same constructs for both concepts. A similar suggestion, based on behavioural semantics with constraints, is elaborated in [Orejas, Nivela 90]. The concept of vertical refinement of [Orejas, Nivela 90] corresponds to our concept of module application.

In the presented approach only few assumptions were made on the underlying logical framework, i.e. the developed concepts on modularity, parameterization and implementation can be "reused" for several particular specification theories. However, it should be clear that our concepts will not have the same power in any particular logical framework. For instance, in a behavioural framework the implementation relation defined here would be much more powerful than in standard theories because in the behavioural case our implementation definition would accept all concrete realizations which satisfy the desired observable behaviour of an abstract specification.

Similarly, the complexity of an appropriate proof theory for proving implementation relations will depend on the particular logical framework in which our approach is used. Since we are especially interested in behavioural specifications (cf. [Bernot, Bidoit 91], [Hennicker 92]) we plan to investigate proof criteria for implementations of modules and of system specifications in the behavioural case. As an appropriate basis we could use e.g. the proof theoretic characterization of behavioural implementations given in [Hennicker 90].

An important further objective of future research is the integration of our concepts into a theory of software reusability. For instance, the approach of [Gaudel, Moineau 88] to software reuse is already based on the stratified loose semantics and hence it would 
be interesting to apply this approach to the case where implementations are treated at the specification level.

Acknowledgement We would like to thank anonymous referees for several helpful comments and suggestions. This work is partially sponsored by the French-German cooperation programme PROCOPE, by the ESPRIT Working Group COMPASS, and by the German BMFT project KORSO.

\section{References}

[Bernot, Bidoit 91] G. Bernot, M. Bidoit: Proving the correctness of algebraically specified software: modularity and observability issues. Proc. AMAST '91, 2nd International Conference on Algebraic Methodology of Software Technology, Techn. Report of the University of Iowa, 1991.

[Bidoit 87] M. Bidoit: The stratified loose approach: A generalization of initial and loose semantics. In: Recent Trends in Data Type Specification, Selected Papers of the 5th Workshop on Specifications of Abstract Data Types, Lecture Notes in Computer Science $332,1-22,1987$.

[Bidoit 89] M. Bidoit: Pluss, un language pour le développement de spécifications algébriques modulaires. These d'État, Université Paris-Sud, 1989.

[Diaconescu et al. 91] R. Diaconescu, J. Goguen, P. Stefaneas: Logical support for modularisation. To appear in: Proc. of Workshop on Logical Frameworks, Edinburgh, 1991.

[Ehrig et al. 89] H. Ehrig, P. Pepper, F. Orejas: On recent trends in algebraic specification. Proc. ICALP' 89 , Lecture Notes in Computer Science 372, 263-288, 1989.

[Ehrig et al. 91] H. Ehrig, M. Baldamus, F. Orejas: New concepts of amalgamation and extension for a general theory of specification. Proc. of the 8th Workshop on Specifications of Abstract Data Types, 1991, to appear in: Lecture Notes in Computer Science.

[Gaudel 92] M. C. Gaudel: Structuring and modularizing algebraic specifications: the PLUSS specification language, evolution and perspectives. Proc. STACS '92, Lecture Notes in Computer Science 577, 3-20, 1992.

[Gaudel, Moineau 88] M. C. Gaudel, Th. Moineau: A theory of software reusability. Proc. ESOP '88. Lecture Notes in Computer Science 300, 115-130, 1988.

[Goguen, Burstall 84] J. A. Goguen, R. M. Burstall: Introducing institutions. Logic of Programs, Lecture Notes in Computer Science 164, 221-255, 1984.

[Hennicker 90] R. Hennicker: Context induction: a proof principle for behavioural abstractions and algebraic implementations. Formal Aspects of Computing 4 (3), 326-345, 1990.

[Hennicker 92] R. Hennicker: Behavioural specification and implementation of modular software systems. Technical Report MIP-9203, University of Passau, 1992.

[Orejas et al. 89] F. Orejas, P. Nivela, H. Ehrig: Semantical constructions for categories of behavioral specifications. Proc. Int. Workshop on Categorical Methods in Computer Science with Applications to Topology. Lecture Notes in Computer Science 393, 1989.

[Orejas, Nivela 90] F. Orejas, M. P. Nivela: Constraints for behavioural specifications. Proc. 7th Workshop on Specification of Abstract Data Types. Lecture Notes in Computer Science $534,220-245,1990$.

[Sannella, Tarlecki 84] D. T. Sannella, A. Tarlecki: Building specifications in an arbitrary institution. Proc. of the Int. Symposium on Semantics of Data Types. Lecture Notes in Computer Science 173, 337-356, 1984.

[Sannella, Tarlecki 87] D. T. Sannella, A. Tarlecki: Toward formal development of programs from algebraic specifications: implementations revisited. Proc. TAPSOFT '87, Lecture Notes in Computer Science 249, 96-110, 1984.

[Sannella et al. 90] D. T. Sannella, A. Tarlecki, S. Sokolowski: Toward formal development of programs from algebraic specifications: parameterisation revisited. Forschungsberichte des Studiengangs Informatik 6/90, Universität Bremen, 1990.

[Schoett 87] O. Schoett: Data abstraction and correctness of modular programming. Ph. D. thesis, CST-42-87, University of Edinburgh, 1987.

[Wirsing 86] M. Wirsing: Structured algebraic specifications: a kernel language. Theoretical Computer Science 42, 123-249, 1986. 